\title{
The Study of Mission Reliability of QRMS Based on the Multistage Markov Process
}

\author{
Liang Liang and Bo Guo \\ School of Information System and Management, National University of Defence Technology, \\ Changsha, Hunan Province, 410073, P.R. China \\ \{Liang Liang, Bo Guo, doulfin\}@gmail.com
}

\begin{abstract}
The iteration is a fundamental characteristic of the complex developing process. The developing mission of new product, during the quick response manufacturing process, will be iterated step by step following certain probability rules at the work nodes. Based on the analysis of the development mission process, the method of the multi-stages, finite state Markov process is presented to describe the system process. The models of the mission reliability for both task stages and system have then been built. Finally, the quantitative methods of mission reliability calculation were explained in details through the analysis of an actual developing process. The methods can also be used to evaluate the development system.
\end{abstract}

Keywords: Quick response manufacturing system(QRMS), Design iteration, Mission reliability.

\section{Introduction}

The Quick Response Manufacturing System (QRMS) is based on the quick response concept with the purpose to quick response to the demand for developing new products and carry out the design and manufacturing process in a very timely manner. The frequent changing of market demand requires higher competence of new product development. On one hand, companies need to analyze its current development \& manufacturing system to estimate, from the perspective of mission reliability, system's ability to finish the task in the required time period. On the other hand, companies may also need to improve on those weaknesses that pertain to the system therefore increase its competence of development.

Iteration and conflict of resource are the most two important factors in an estimation of the mission reliability for a QRMS. Iteration is a basic characteristic of the complex development process ${ }^{[1,2]}$. The iteration processes means rework and improve on previous work and that always leads to risk of scheduling and repeated planning and eventually make the whole developing process delayed. The fast changing demand of market gets the QRMS overloaded and that means a task always has to queue in the process due to the lack of resource. Hence, the task cannot be finished on time and companies will suffer loss.

Currently, Design structure Matrix ${ }^{[3]}$ (DSM) are widely used for the time evaluation of product development process. DSM has been extended to the numerical DSM, 
which can further describe the iteration relation among different developing processes. In DSM, probability of rework indicates the possible rework of the whole developing process. However, the scenario derived from DSM that the probability of rework is a constant does not reflect the actual operating rule. Therefore, this paper extends the probability of rework scenario by taking the changing probability of rework during different task developing stages into account.

The conflict of recourse in the developing process can be better reflected using the model of queuing network. Meanwhile, the transferring matrix of queuing network can be related to the matrix derived from DSM. Hence, the queuing network model has greater applicability as during the developing process. The paper analyzes the task developing process of quick response manufacturing system and describes the dynamic probability of rework by adopting multi-stages, finite states Markov process. The paper finally figures out the analytical model and arithmetic for task reliability calculation by analyzing and modelling those probabilities using queuing network. The following cases further show how arithmetic works.

\section{Problem Formulation}

Suppose there are $M$ nodes in a QRMS and each division has one workgroup. The service time for each workgroup follows exponential distributions with parameter $\mu_{i}$ $(i=1,2,3 \ldots N)$. Additional predetermined assumptions are:

1. Those tasks that have been undertaken by the system are homogeneous. The arrival process is Poisson with arrival rate $\lambda$. The tasks arrive at the first node from outside and finish all the service work in the order of the nodes. When task arrives, the idle workgroup reacts immediately while others keep waiting on a queue. Services at all nodes are independent and the number of queues at each node is limitless.

2. In a task developing process, the reworks exist and are allocated in each node following certain probability. When a task is first finished at node $i$, it can only be returned to node $(1,2, \cdots, i-1)$ if any rework occurred.

3. Followed by the proceeding of a task among nodes, the probability of rework will change because new information arrives.

\section{System Modelling and Analysis}

\subsection{Model Definition}

\section{Definition 1:}

Set $A=\left\{t_{i}^{(1)}, i=1,2,3 \ldots N\right\}, t_{i}^{(1)}$ is referred to as the point of time when the task first arrives (be proceeded) at node $i$. Set $t_{1}^{(1)}=0$, and $t_{N+1}^{(1)}$ is referred to as the point of time when task has been finished. 
From the hypothesis 1 , if the points of time $t_{1}^{(1)}, t_{2}^{(1)}, \ldots, t_{N+1}^{(1)}$ all exist, then:

$$
t_{1}^{(1)} \leq t_{2}^{(1)} \leq \ldots \leq t_{N+1}^{(1)}
$$

\section{Definition 2:}

The stage $I$ of a task is referred to as the process of a task from node $i$ to node $i+1$, which can defined as the period $\left\{\zeta_{i} \mid t_{i}^{(1)} \leq \zeta_{i}<t_{i+1}^{(1)}, i=1,2,3 \ldots N\right\}$.

\section{Definition 3:}

Set $X_{I}=t_{i+1}^{(1)}-t_{i}^{(1)}, i=1,2, \cdots, N, T_{I}$ indicates the given time to finish the task at stage $I$. The mission reliability of stage $I$ can be defined as follows:

$$
R_{I}\left(X_{I}\right)=P\left[X_{I} \leq T_{I}\right]
$$

\section{Definition 4:}

Set $X=t_{N+1}^{(1)}=\sum_{I=1}^{N} X_{I}, T$ indicates the given time to finish the task. The system's mission reliability can be defined as:

$$
R(X)=P[X \leq T]
$$

\section{Definition 5:}

The task will enter the second node directly after reaching the first node at first time and being finished at this node. That is, there is no rework in the first stage, symbolized as $\mathbf{P}_{1}=[0]$. While in the stage $I(I=2, \cdots, M)$, the rework at different nodes can be illustrated by the following matrix:

$$
\mathbf{P}_{I}=\left[\begin{array}{ccc}
r_{11}(I) & \ldots & r_{1 i}(I) \\
\vdots & \ddots & \vdots \\
r_{i 1}(I) & \cdots & r_{i i}(I)
\end{array}\right]
$$

$r_{i j}(I)$ : The probability of task being reworked from the first node $i$ to node $j$ $(0<i, j \leq I)$ when task has been processed in stage $I$.

\subsection{The Single Task Process Modelling}

The development process is shown in Fig. 1. It is known from the definition that under the system discussed in this paper, the rework of a task can only happen among the nodes which have been serviced already. Further, the probability of rework is changing upon the task being processed forward. This paper then puts forward a measure called Multistage, Finite-state, absorbing Markov chains for system modelling and analyzing. 


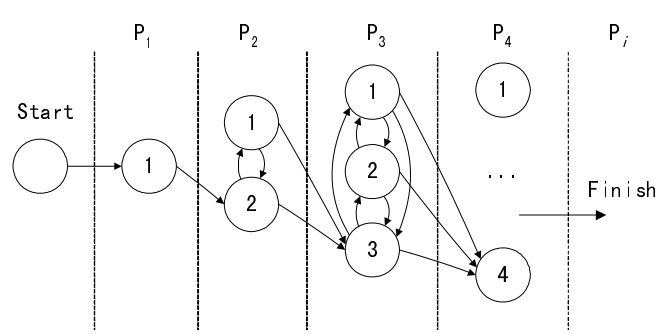

Fig. 1. The development process based on dynamic probability of rework

For single task, when it has been processed to stage $I$, at the observation time $t_{i}^{(1)}$, task reaches node $i$ at first time. After that, a virtual absorbing node $(i+1)^{\prime}$ will be constructed, that is $r_{(i+1)^{\prime} j}=0, j=1,2, \cdots, i$, set:

$$
\left\{\begin{array}{l}
r_{j}(i+1)^{\prime}=r_{j(i+1)} \\
t_{(i+1)^{\prime}}^{(1)}=t_{(i+1)}^{(1)}
\end{array}\right.
$$

given that the matrix $\boldsymbol{\beta}_{I}=\left(\beta_{i j}\right)=\left(\mathbf{P}_{I}-\mathbf{e}\right)$ is invertible square matrix. (Throughout this paper, e will denote the column vector with all components equal to one, whose length is determined by the context in which it appears.), Without regard to the waiting time taken in the queue at nodes, it can be shown from Eq. (3) that the finitestate, absorbing Markov Chains has been constructed during the process of the task from node $i$ to note $(i+1)^{\prime}$. The initial state of the process is $\left(\boldsymbol{\alpha}_{I}, \alpha_{I+1}\right)$, where $\boldsymbol{\alpha}_{I}=(0, \cdots, 0,1)_{1 \times I}$. The set of states is $\left(1, \ldots, i,(i+1)^{\prime}\right)$. Among that the state $(1,2, \cdots, i)$ are all transient, state $(i+1)^{\prime}$ is absorbing. Before the process absorbed to the state $(i+1)^{\prime}$, the mean visit times to each node can be described as the vector ${ }^{[4]}$ :

$$
\mathbf{K}_{I}=\left(k_{I}(1), \cdots, k_{I}(j), \cdots, k_{I}(i)\right)=\boldsymbol{\alpha}_{I} \int_{0}^{\infty} \exp \left[\left(\mathbf{P}_{I}-\mathbf{e}\right) x\right] d x=-\boldsymbol{\alpha}_{I} \boldsymbol{\beta}_{I}^{-1}
$$

\subsection{Steady-State System Development Process Model}

\subsubsection{Steady-State Mean Arrival Rate of Each Node}

From assumption we can know that the arrivals of tasks follow independent, exponentially distributed interarrival times with rate $\lambda$. The task service time at each node also follows exponentially distributed interarrival times with rate $\mu_{i}$. Under steady state, every node in the queuing network can be viewed as a $M / M / 1$ queuing system. Thus, the arrival rate of a task entering quick response manufacturing system, under steady state, must equalize its departure rate. Assume that total mean arrival 
rate (the sum of the arrival rate from outside and those from other nodes to node $i$ ) is $\Lambda_{i}$ at node $i$, then :

Theorem 1. under steady state, the total mean arrival rate of each node is

$$
\Lambda_{j}=\lambda \sum_{I=1}^{N} k_{I}(j) \quad(j=1,2, \cdots, N)
$$

Proof: Under steady state, the jobs at node $j$ come from every stage in the whole task process. From Eq.(4), in the task stage $I$, the mean times for task visiting node $j(j=1,2, \cdots, i)$ is $k_{I}(j)$, therefore, under steady state, the sum of the mean times for task to come from every stage visiting node $j$ is $\sum_{I=1}^{N} k_{I}(j)$.

From model assume: the task stage is the sequence, the task from outside to the first node in the system follows the Poisson process with parameters $\lambda$, and the other nodes haven't got any input from outside. Then the steady-state overall arrival rate at node $j(j=1,2, \cdots, N)$ can be written as:

$$
\Lambda_{j}=\lambda \sum_{I=1}^{N} k_{I}(j) \quad(j=1,2, \cdots, N)
$$

The theorem has then been proved.

\subsubsection{Steady-State Response Time Distribution $W_{i}(t)$ of Node $i$}

When the utilization $\rho_{i}$ of the node $i$ meets:

$$
\rho_{i}=\frac{\Lambda_{i}}{u_{i}}<1 \quad(i=1,2, \cdots, N)
$$

queueing network will leave the steady-state distribution, which the marginal queue length distribution of every node at the queueing networks is the same as that of the $M / M / 1$ queueing system ${ }^{[5]}$. Then the distribution of steady-state response time (the sum of the waiting time and the server time) at node $i(i=1,2, \cdots, N)$ is:

$$
W_{i}(t)=P\left[V_{i} \leq t\right]=\mathrm{E}\left[P\left[V_{i} \leq t\right] \mid N=n_{i}\right]=1-e^{-\left(\mu_{i}-\Lambda_{i}\right) t}, \quad t \geq 0
$$

From Eq.(7), the steady-state distribution at node $i$ is exponentially distribution with parameters:

$$
\lambda_{i}=\mu_{i}-\Lambda_{i}
$$

\subsection{The System Modelling for Mission Reliability}

\subsubsection{Mission Reliability Model of the Task Stage}

Theorem 2. set $\lambda_{i}=\left(\lambda_{1}, \lambda_{2}, \cdots \lambda_{i}\right)_{1 \times i}, i=1,2, \cdots, M$, under steady state, mission reliability at task stage $I$ : 


$$
R_{I}(t)=1-\boldsymbol{\alpha}_{I} \exp \left[\mathbf{T}_{I} t\right] \mathbf{e}, t \geq 0
$$

Where, $\boldsymbol{\alpha}_{I}=(0, \cdots, 0,1)_{1 \mathrm{x} I}, \mathbf{T}_{I}=\left(T_{k j}\right)=\left\{\lambda_{i} \beta_{i j}\right\}, \beta_{i j}$ is the matrix element defined by the Eq.(4).

Proof: From Eq.(8), the steady-state distribution at every node is exponentially distribution. Therefore, in stage $I$, the process constructed in the 2.2 section was a finitestate Markov process with generator:

$$
\mathrm{Q}_{I}=\left(\begin{array}{cc}
\mathbf{T}_{I} & \mathbf{T}_{I}^{0} \\
\mathbf{0} & 0
\end{array}\right)
$$

The matrix $\mathbf{T}_{I}=\left(T_{k j}\right)=\left\{\lambda_{i} \beta_{i j}\right\}$ of order $I$ is non-singular. It has negative diagonal elements and nonnegative off-diagonal elements and satisfies $\mathbf{T}_{I} \mathbf{e}+\mathbf{T}_{I}{ }^{0}=\mathbf{0}$. The initial state of the process is $\left(\boldsymbol{\alpha}_{I}, \alpha_{I+1}\right)$, where $\boldsymbol{\alpha}_{I}=(0, \cdots, 0,1)_{1 \mathrm{x} I}$. Then the absorbing time distribution of Markov process follows the Phase-Type(PH) distribution. The distribution function is :

$$
F_{I}(t)=P\left[t \leq T_{I}\right]=1-\boldsymbol{\alpha}_{I} \exp \left[\mathbf{T}_{I} t\right] \mathbf{e}, t \geq 0
$$

Where, $T_{I}$ was the given time to finish the task at stage $I$.

From Definition 3, it is proved that $R_{I}(t)=F_{I}(t)$.

\subsubsection{Mission Reliability Model of the System}

From Definition 3, $X_{I}(i=1,2, \cdots, N)$ was defined as the actual task serving time at stage $I$, and based on the system hypothesis, $X_{I}$ is non-negative independent random variables, and the total time of a task $X=\sum_{I=1}^{N} X_{I}$, then the total service time distribution of a task is the convolution of the service time distribution of each task stage.

The convolution of the limited number of PH distribution is also the $\mathrm{PH}$ distribution $^{[4]}$. It is obviously that it is the finite process for a task in QRMS. The total service time distribution of a task also follows the PH distribution denoted as $(\boldsymbol{\gamma}, \mathbf{L})$, then the mission reliability of the system

$$
R(t)=P[t \leq T]=1-\gamma \exp [\mathbf{L} t] \mathbf{e}, t \geq 0
$$

Where $T$ is the given time to finish the task

\section{Case Study}

The key accessory design for an aero engine makes up of five working nodes: unit design, finite analysis, artwork design, mould design, and quick-to-mockup. The task 
departs the system after going through above five nodes one by one. The arrivals of tasks follow the exponential distribution with parameters $\lambda=0.007$.

Table 1. The parameter of the nodes

\begin{tabular}{llllll}
\hline Nodes_No. & 1 & 2 & 3 & 4 & 5 \\
\hline$\mu_{i}$ & 0.0240 & 0.0310 & 0.0320 & 0.0252 & 0.0350 \\
\hline
\end{tabular}

The rework probability matrixes for each stage are as follows:

$$
\begin{aligned}
& \mathrm{P}_{1}=[0] \quad \mathrm{P}_{2}=\left[\begin{array}{cc}
0 & 0.95 \\
0.15 & 0
\end{array}\right] \quad \mathrm{P}_{3}=\left[\begin{array}{ccc}
0 & 0.90 & 0 \\
0.12 & 0 & 0.8 \\
0.3 & 0.2 & 0
\end{array}\right] \\
& \mathrm{P}_{4}=\left[\begin{array}{cccc}
0 & 0.85 & 0 & 0 \\
0.1 & 0 & 0.7 & 0 \\
0.25 & 0.15 & 0 & 0.5 \\
0.1 & 0 & 0 & 0
\end{array}\right] \quad \mathrm{P}_{5}=\left[\begin{array}{ccccc}
0 & 0.7 & 0 & 0 & 0 \\
0.05 & 0 & 0.6 & 0 & 0 \\
0.2 & 0.1 & 0 & 0.4 & 0 \\
0.05 & 0 & 0 & 0 & 0.8 \\
0 & 0 & 0 & 0.1 & 0
\end{array}\right]
\end{aligned}
$$

Detail calculation result : Based on Eq. (5) and Eq. (8), the total mean arrival rate $\Lambda_{i}$ and the mean response time $1 / \lambda_{i}$ can be shown in Table 2. Based on Eq. (9) \& (10), the mission reliability curves for each stage and the whole system can be showed in Fig. 2 .

Table 2. Steady-state arrival rate $\Lambda_{i}$ and mean serving time $1 / \lambda_{i}$

\begin{tabular}{llllll}
\hline node_no & 1 & 2 & 3 & 4 & 5 \\
\hline$\Lambda_{i}$ & 0.0137 & 0.0155 & 0.0128 & 0.0081 & 0.0076 \\
\hline $1 / \lambda_{i}$ & 96.674 & 64.575 & 52.029 & 58.477 & 36.517 \\
\hline
\end{tabular}

From Fig. 2, it is known that under the same requirement for mission reliability, the task in the third stage takes the longest time to be finished, that is, it takes the longest time for a task being processed from node 3 to node 4 . Therefore, the third stage is the bottleneck for the whole mission. Table 2 shows the average waiting time at node 3 is $52.029 \mathrm{~h}$. Such long time being taken is mainly due to the reworks caused by design iteration. Hence the designer can consider some measures to reduce the design failure and improve the level of system's mission reliability level. Possible measures are: the increase of design quality control; more training for designing staff, and so on. 


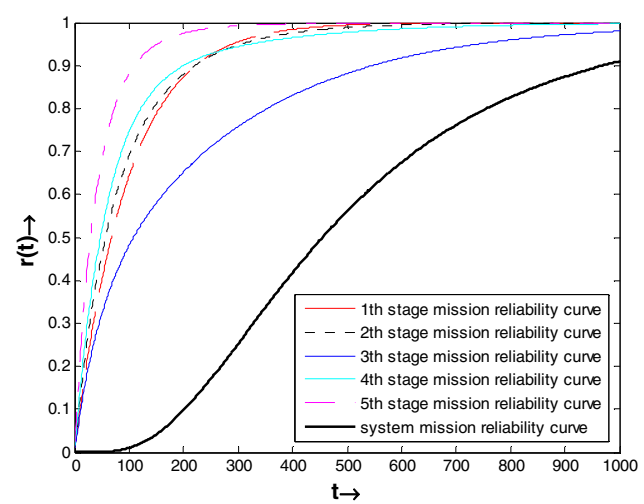

Fig. 2. Mission reliability curve of each stage and system

\section{Conclusion}

By analyzing the mission reliability for the QRMS, a model has been built to reflect such mission reliability under dynamic iteration and conflict of resource. Consequently, a quantitative solution has been given to calculate those mission reliabilities for each stage and for the whole system. Both the model and solution give an effective tool to evaluate the mission reliability of quick response manufacturing. They also facilitate the discovery of system's weaknesses and give great help to improve those weaknesses.

\section{References}

1. Eppinger, S., Whitney, D., Smith, R., Gebala, D. A Model-Based Method for Organizing Tasks in Product Development[J]. Research in Engineering Design. 1994.6: pp.1-13.

2. Cho, S.-H., Eppinger, S. D. A simulation-based process model for managing complex design projects[J]. IEEE Transactions on Engineering Management. 2005(52).3: pp.316-328.

3. Peter, B., LuhFeng, L., Bryan, M. Scheduling of Design Projects with Uncertain Number of Iterations[J]. European Journal of Operational Research. 1999(113).3: pp.575--592.

4. Naishuo, T., Dequan, Y. Quasi Birth-and-death Process and Matrix--Geometric Solutions(in Chinese)[M]. Beijing: Science Press. 2002.

5. Bolch, G., Greiner, S., Meer, H. d., Trivedi, K. S. Queueing Networks and Markov Chains-Modeling and Performance Evaluation with Computer Science Applications[M]. Hoboken: John Wiley \& Sons, Inc. 2006. 